\title{
Caracterización fisicoquímica del aceite de milpesos y evaluación de alternativas tecnológicas para mejorar su aprovechamiento en las comunidades de La Plata, Málaga, Buenaventura
}

\section{Physicochemical characterization of mil pesos oil and evaluation of technological alternatives to improve its use in communities of La Plata, Málaga, Buenaventura}

\author{
Nixon Arboleda Montaño, MSc* \\ RESUMEN
}

El presente artículo registra los principales resultados del estudio de alternativas tecnológicas sostenibles para mejorar los procesos de aprovechamiento de las palmas de milpesos en la comunidad de La Plata, Bahía Málaga, Buenaventura, presentando en primer lugar los principales usos que en la zona estudiada se le da a la palma de milpesos; se establecen las características y usos del aceite de milpesos, tratando fundamentalmente lo relacionado con su composición de los ácidos grasos y sus propiedades físico-químicas; se hace mención al proceso de extracción tradicional de aceite de palma de milpesos, desarrollando sus diez fases; además, se presenta una evaluación de la técnica tradicional de extracción de aceite. Se comparan los métodos físico (con despulpado mecánico y extracción de aceite por prensado) y químico (utilizando solvente), para luego realizar una evaluación teniendo en cuenta los factores ambiental, socio-cultural, económico y técnico-operativo; finalmente, se desarrolla el método físico (con despulpado mecánico y extracción de aceite por prensado) porque de acuerdo con la evaluación realizada se determinó que es el más apropiado para mejorar el sistema tradicional de extracción de aceite en el área de estudio.

Palabras clave: Palma de milpesos; Método tradicional de extracción de aceite; Bahía Málaga; Tecnologías sostenibles; Método físico; Método químico.

\begin{abstract}
This article presents the main results of the study of alternative sustainable technologies to improve the processes of exploitation of the palma de mil pesos in the community of La Plata, Bahía Málaga, Buenaventura, presenting first the main uses in the study area is give the palma de milpesos; then establishing the characteristics and uses milpesos oil, mainly trying to do with its fatty acid composition and physicochemical properties, in another separate mention is made of traditional extraction process aceite de milpesos, to develop its 10 phases also provides an assessment of the traditional technique of oil extraction, by a quarter point method compares the physical (mechanical pulping and pressing oil extraction), and chemical method (using solvent), and then make an assessment taking into account environmental factors, socio-cultural, economic and technical-operational, and finally, develop the physical method (with mechanical pulping and pressing oil extraction) because of according to the
\end{abstract}

* Master en Proyectos Ambientales, Especialista en Gestión Ambiental, Administrador Ambiental, Investigador Instituto de Investigaciones Ambientales del Pacífico (IIAP), Quibdó, Colombia. e-mail: nixon@utp.edu.co Recibido: 11 de agosto de 2011 Aceptado: 3 de noviembre de 2011 
evaluation it was determined that it is more appropriate to improve the traditional system of oil extraction in the study area.

Keywords: Palma de milpesos; Traditional method of extracting oil; Bahía Málaga; Sustainable technologies; Physical method; Chemical method.

\section{INTRODUCCIÓN}

La alta diversidad biológica del Chocó Biogeográfico le permite contar con grupos biológicos y especies de interés especial, bien sea por caracteres inherentes a su valor ecológico o por razones de su potencialidad para muy diversos usos, tal es el caso de la palma de milpesos o seje (Oenocarpus bataua), a partir de la cual se pueden obtener alimentos, medicina, combustible, artesanías, materiales para construcción, no siendo menos importante el valor cultural que representa para las comunidades.

En este sentido, las comunidades estudiadas han apropiado diferentes usos y sistemas de aprovechamiento de la palma de milpesos, algunos de los cuales se hace necesario mejorar mediante procesos más eficientes, que generen mayores beneficios comunitarios, objetivo fundamental de la investigación adelantada, además de identificar los principales usos de la palma y de su aceite en la comunidad, determinar las propiedades físicas y químicas del aceite, describir el proceso de extracción tradicional y desarrollar un método de aprovechamiento que tome en cuenta criterios ambientales, socio-culturales, económicos y técnico-operativos.

Localización del estudio. Buenaventura es un puerto de rada abierta, con protección natural dentro de una bahía interior abrigada. El territorio municipal es el de mayor extensión en el departamento de Valle del Cauca y está comprendido desde las orillas del océano Pacífico, hasta las cumbres de la cordillera Occidental, en el sector de los Farallones de Cali, que le sirven de límite con los municipios de Jamundí y Cali. Limita por el norte con el departamento del Chocó, por el oriente con los municipios de Jamundí, Cali, Dagua y Calima Darién, por el sur con el departamento del Cauca y por el occidente con el océano Pacífico.

Por su parte, Bahía Málaga revela una topografía irregular, diferenciada por una zona este y sureste poco profunda, en la que sobresalen a manera de islotes (La Plata) algunas mesetas de abrasión colonizadas por manglares, y con promontorios rocosos que constituyen obstáculos a la navegación. Al oeste y noroeste de la bahía se halla la formación de un canal natural profundo (25-30 m), que conduce las mareas entrantes y salientes de Bahía Málaga y que a su vez conforman el patrón de corrientes predominante.

Respecto a su biodiversidad florística, se han reportado 400 especies arbóreas, existen cuatro especies de mangle; 10 de algas rojas; seis de algas verdes y dos de algas pardas, y palmas como el milpesos, convirtiéndose esta última en el objetivo de la presente investigación.

\section{METODOLOGÍA}

Recolección de la información. Para determinar los usos y sistemas de aprovechamiento que la comunidad en estudio hace de la palma de milpesos, se procedió de la siguiente forma:

Caracterización de los sistemas de aprovechamiento. Mediante un trabajo de campo de cinco meses en la zona de estudio, se recolectó información utilizando instrumentos tales como encuestas, entrevistas, seguimientos a las formas de aprovechamiento, entre otras. El análisis de la información se efectuó mediante el procesamiento de los datos en paquetes estadísticos y su correlación de la información secundaria, proveniente de estudios relacionados con el tema.

Estudio de los procesos artesanales de extracción y caracterización fisicoquímica del fruto y el aceite

Caracterización fisicoquímica del aceite. Los análisis fisicoquímicos del aceite de milpesos se realizaron en los laboratorios de química de la Universidad del Valle, siguiendo normas ICONTEC. La composición de ácidos grasos se determinó por medio de la cromatografía de gases para la muestra obtenida por el método artesanal.

Propiedades físicas, componentes y análisis proximal de los frutos de milpesos. Para el análisis de los frutos de milpesos se separaron sus tres componentes: cáscara, pulpa y semilla. El procedimiento fue el siguiente:

a) Se seleccionaron, de áreas representativas, seis palmeras con abundantes frutos en estado de madurez; de cada palmera de cosecharon los frutos y se tomaron $4 \mathrm{~kg}$ que después de ser lavados cuidadosamente se congelaron hasta su posterior transporte a laboratorio. Cada grupo constituyó una muestra.

b) De cada muestra se tomaron al azar 10 frutos, se determinó su peso, su longitud y su anchura, con el uso de balanza electrónica.

c) Se separó con cuchilla la pulpa (mesocarpio más epicarpio) de la semilla. A las semillas (endocarpio) se les pesó y midió largo y ancho.

d) Se separó el mesocarpio del epicarpio con cuchilla y se hallaron los pesos.

e) Se repitió el procedimiento para los sesenta frutos, agrupando los datos en seis grupos de 10 datos cada uno.

Los datos se procesaron en el programa Excel, obteniendo como resultado las correspondientes propiedades físicas como peso y medidas y el porcentaje de componentes.

Método para hallar densidad real, densidad aparente y porosidad. Para determinar estas propiedades, se realizó el 


\section{Bioetnia Volumen 8 No 2 (julio-diciembre), 2011}

siguiente procedimiento:

- Se seleccionaron tres grupos de frutos con pesos entre 10 $\mathrm{kg}$ y $15 \mathrm{~kg}$.

- Se determinó el peso exacto de un grupo de frutos (P).

- Se vaciaron los frutos en un recipiente cuadrangular de pared rígida acomodados adecuadamente.

- Se midió el volumen ocupado por los frutos en el recipiente (V1).

- Se determinó la densidad aparente (P/V1).

- Se adicionó agua desde un recipiente calibrado hasta el nivel restante con los frutos.

- Se determinó el volumen de agua adicionado (V2).

- Se halló la densidad real como (P/(V1-V2).

- Se determinó la porosidad como (V2/V1)*100.

- Se obtuvo el volumen unitario como (V1-V2)/n, donde $n$ es el número de frutos en el recipiente.

- Se repitió el procedimiento para los otros dos grupos de frutos.

Caracterización del método artesanal de producción de aceite. Para determinar las características, etapas y cantidades involucradas en el proceso de extracción de aceite de palma en Bahía Málaga se realizó el siguiente procedimiento:

- Se dialogó con las familias más dedicadas a la extracción de aceite.

- Se asistió a algunos eventos de extracción para definir y caracterizar el método de extracción artesanal. Se tomaron datos como duración de las etapas del proceso, cantidad de trabajo empleado, frutos en el proceso, cantidad de agua utilizada, leña empleada resultante del proceso, materiales y herramientas, y aceite final obtenido.

- Se censó la producción de aceite por medio de encuestas a productores.

Procedimiento para hallar los parámetros del método artesanal. Se realizó el método artesanal con las comunidades de estudio para cinco repeticiones. El peso del aceite se determinó de manera indirecta, por medio de su volumen. La duración de las diversas labores (cocinado y lavado), así como el trabajo se midieron con cronómetro digital. El trabajo medido en horas/persona se determinó para las labores que exigen mayor esfuerzo físico, como lavado, macerado y colado. Para otras labores complementarias se estimó un tiempo de acuerdo con las observaciones. La leña utilizada fue previamente pesada y luego de la combustión los sobrantes también se pesaron para hallar por diferencia la cantidad usada. El agua que se usó en las labores de extracción de aceite se midió con recipientes calibrados de diez litros de capacidad.

Gestión de la información. La gestión de la información secundaria se realizó con base en el método RACEER propuesto por Hurtado de Barrera (2000, 2002), mediante el cual se efectúa el proceso de documentación, es decir, el cumplimiento de las fases heurística y hermenéutica que conducen al desarrollo y la conformación escrita de la ya mencionada base teórica. RACEER es una sigla que contiene las iniciales de cada uno de los procedimientos empleados en todo proceso de documentación tanto en un proceso investigativo como en el desarrollo de diferentes textos como monografías, ensayos, artículos científicos y periodísticos.

Recopilación de la información.

Almacenamiento en unidades de información.

Categorización de unidades temáticas.

Elaboración del esquema conceptual o de contenidos de la producción escrita.

Enlace y coordinación temática que permita mantener la ilación y coherencia de los diferentes contenidos.

Redacción del texto final.

De acuerdo con lo anterior, en esta etapa se realizaron visitas a las entidades del orden local, regional y nacional como CVC, laboratorio de la Universidad del valle, UNIPACIFICO, Universidad Nacional sede Palmira, entre otras. También se tuvo en cuenta información secundaria de fuentes bibliográficas acerca de otros procesos de investigación bibliométrica, de cienciometría e informetría. La gestión de la información permitió evaluar las alternativas tecnológicas identificadas, teniendo en cuenta aspectos ambientales, socioculturales, económicos, técnicos y operativos.

\section{RESULTADOS}

Usos asignados a la palma de milpesos. Los resultados de esta investigación muestran que la palma de milpesos es uno de los recursos vegetales más útiles para la comunidad de Bahía Málaga, porque de una u otra manera le proporciona alimento, vivienda, medicina, combustible, artesanía, materiales para construcción y aportes culturales.

En la Figura 1, se presentan los resultados de los usos asignados a la palma de milpesos. De acuerdo con los usos asignados la alimentación es el más representativo y dentro de este la elaboración de aceite, por lo que se hace necesario implementar estrategias que permitan mejorar el aprovechamiento que se hace actualmente de esta palma.

Características y usos del aceite de milpesos. En la Tabla 1 se muestra la composición de ácidos grasos del aceite de milpesos en la zona de estudio, además de otros resultados obtenidos por diferentes autores con el mismo aceite, de diversos sitios. Se incluye un análisis de aceite de oliva, para permitir la comparación con el aceite de milpesos. Es de resaltar el elevado porcentaje del ácido oleico (82\%), que es hasta ahora el aceite que tiene la mayor concentración de un solo ácido graso.

La fracción saturada del aceite de milpesos está representada por los ácidos grasos láurico, mirístico, palmítico y esteárico, de 


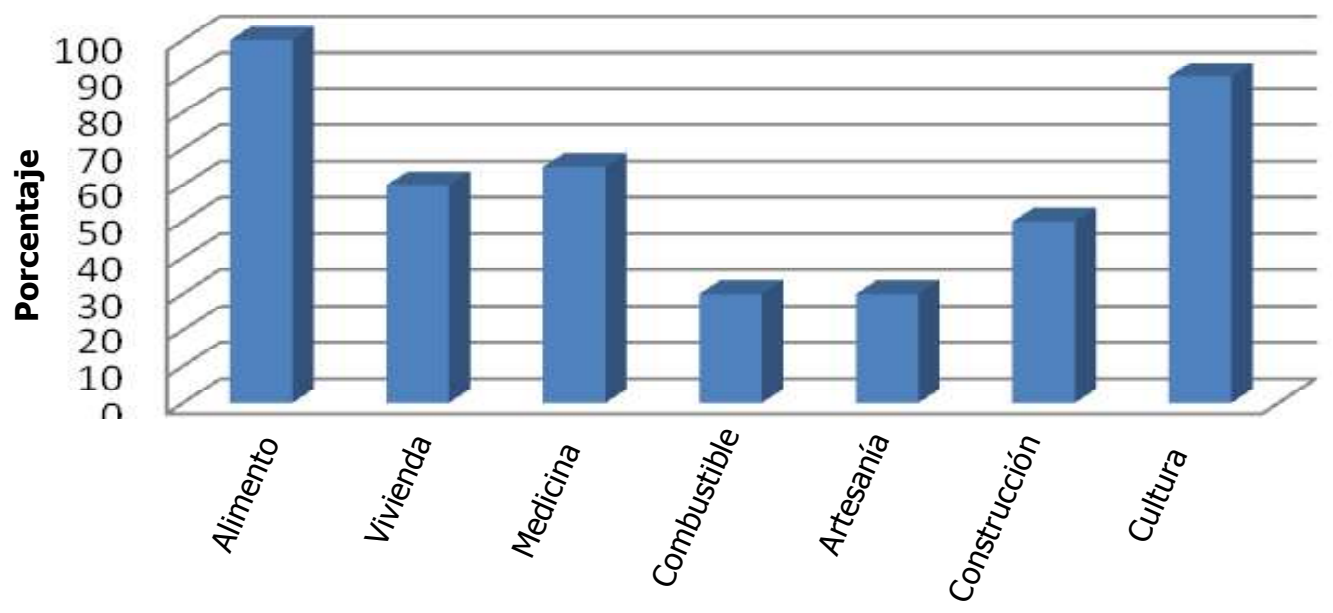

Figura 1. Usos asignados a la palma de milpesos

Tabla 1

Composición de los ácidos grasos del aceite de milpesos

\begin{tabular}{lc}
\hline Ácido graso & Valoración (\%) \\
\hline Laúrico & 0,1 \\
Mirístico & 0,4 \\
Palmítico & 10,5 \\
Palmitoleico & 1,4 \\
Esteárico & 1,5 \\
Oléico & 82,0 \\
Linoléico & 3,3 \\
Linolénico & 0,6 \\
Arachidico & 0,1 \\
Erucico & 0,2 \\
\hline
\end{tabular}

$12,5 \%$ para las muestras de este trabajo. El ácido saturado dominante es el palmítico con $10,5 \%$ de la muestra y $84 \%$ de la fracción saturada. Laparte monoinsaturada delaceite de milpesos está representada por los ácidos palmitoléico y oléico, con un porcentaje un poco mayor de $83 \%$, predominando en la fracción el ácido oléico (98\%). Los ácidos grasos poliinsaturados del aceite de milpesos son el linoléico y linolénico, con una fracción de $3,9 \%$ siendo mayoritario el linoléico con el $83 \%$ de la fracción. En cuanto a la composición de ácidos grasos, el aceite de milpesos y el aceite de oliva son similares, con una pequeña diferencia en cuanto al contenido de ácido linoléico, porque el aceite de oliva contiene dos veces más que el aceite de milpesos.

Índices. En la Tabla 2 se muestran los diferentes índices fisicoquímicos que cualifican al aceite de milpesos. El índice de yodo (91) refleja la insaturación del aceite. La densidad de aceite $(0,910 \mathrm{~g} / \mathrm{ml})$ y el índice de refracción $(1,466)$ son
Tabla 2

Índices característicos del aceite de milpesos

\begin{tabular}{lc}
\hline \multicolumn{1}{c}{ Índice } & Valores \\
\hline Densidad $25^{\circ} \mathrm{C}$ & $0,910 \mathrm{~g} / \mathrm{ml}$ \\
Viscosidad & $76,832(\mathrm{C}$. Stokes $)$ \\
\hline Tensión superficial & $34,5\left(\right.$ Dinas $\left./ \mathrm{cm}^{2}\right)$ \\
Índice de refracción $25^{\circ} \mathrm{C}$ & 1,466 \\
\hline Punto de fusión & $8^{\circ} \mathrm{C}$ \\
Punto de humo & $185^{\circ} \mathrm{C}$ \\
\hline Índice de yodo & $91,0 \mathrm{~g} \mathrm{I} 2 / 100 \mathrm{ml}$ \\
Índice de saponificación & $190 \mathrm{mg} / \mathrm{ml} \mathrm{KOH}$ \\
\hline Materia insaponificable & $0,5 \%$ \\
Índice de acidez & $1,5 \mathrm{mg} / \mathrm{ml} \mathrm{KOH}$ \\
\hline Índice de peróxido & $3,3 \mathrm{meq} \mathrm{O} / \mathrm{Kg}$ \\
Índice de esteres & $188,5 \mathrm{mg} / \mathrm{ml} \mathrm{KOH}$ \\
Rancidez de Kreibs & $\mathrm{Negativa}$ \\
Ácidos grasos libres & $1 \%$ \\
\hline
\end{tabular}

propiedades físicas, fácilmente medibles y datos muy importantes, pues permiten evitar falsificaciones. El índice de saponificación (190) y el ácido graso predominante (oleico) caracterizan la buena disponibilidad del aceite de milpesos para convertirse en jabones suaves y traslucidos. La materia insaponificable es baja $(0,5 \%)$, refleja el método de extracción del aceite.

El índice de acidez (1,5), el contenido de ácidos libres (1\%) y el índice de peróxidos reflejan el estado de conservación del aceite, su baja oxidación y desdoblamiento de triglicéridos, también reflejan las deficiencias del método de extracción. Aunque están bajos, se pueden obtener estos 


\section{Bioetnia Volumen 8 No 2 (julio-diciembre), 2011}

Tabla 3

\section{Comparación de seis muestras de milpesos}

\begin{tabular}{|c|c|c|c|c|}
\hline Análisis & Unidades & Promedio & Desviación & Variación \\
\hline \multirow[t]{2}{*}{ Peso fruto } & Inicial (g) & 15,47 & 1,24 & 1,59 \\
\hline & Final $(g)^{*}$ & 14,96 & 1,26 & 1,62 \\
\hline \multirow[t]{2}{*}{ Peso semilla } & (g) & 8,99 & 0,95 & 0,92 \\
\hline & (\%) del fruto & 59,79 & 1,93 & 3,89 \\
\hline \multirow[t]{2}{*}{ Peso cascara } & Final $(\mathrm{g})$ & 3,03 & 0,24 & 0,06 \\
\hline & $(\%)$ & 20,39 & 0,88 & 0,82 \\
\hline \multirow[t]{2}{*}{ Peso pulpa } & (g) & 2,95 & 0,20 & 0,05 \\
\hline & (\%) del fruto & 19,82 & 1,61 & 2,71 \\
\hline \multirow[t]{2}{*}{ Medidas fruto } & Largo cm & 3,96 & 0,12 & 0,01 \\
\hline & Ancho cm & 2,48 & 0,06 & 0,00 \\
\hline \multicolumn{5}{|l|}{ Medidas } \\
\hline semillas & $\begin{array}{l}\text { Largo cm } \\
\text { Ancho cm }\end{array}$ & $\begin{array}{l}3,54 \\
1,99\end{array}$ & $\begin{array}{l}0,12 \\
0,09\end{array}$ & $\begin{array}{l}0,02 \\
0,01\end{array}$ \\
\hline Esfericidad & $\%$ & 73,36 & 1,32 & 1,92 \\
\hline $\begin{array}{l}\text { Densidad } \\
\text { aparente }\end{array}$ & $\mathrm{g} / \mathrm{cm}^{3}$ & 0,724 & 0,018 & 0,0003 \\
\hline Densidad real & $\mathrm{g} / \mathrm{cm}^{3}$ & 1,1 & 0,017 & 0,0003 \\
\hline Porosidad & $\%$ & 35,21 & 1,536 & 2,36 \\
\hline $\begin{array}{l}\text { Volumen } \\
\text { unitario }\end{array}$ & $\mathrm{cm}^{3}$ & 14,06 & 0,48 & 1,63 \\
\hline $\begin{array}{l}\text { Ángulo de } \\
\text { reposo }\end{array}$ & grados & 34 & 1,724 & 2,85 \\
\hline $\begin{array}{l}\text { Ángulo de } \\
\text { fricción }\end{array}$ & grados & 18 & 1,915 & 3,22 \\
\hline
\end{tabular}

* Peso del fruto como suma de sus componentes

Tabla 4

Análisis proximal de los componentes $y$ el fruto milpesos

\begin{tabular}{lcccc}
\hline Análisis & Cáscara & Pulpa & Semilla & Fruto \\
\hline Composición (\%) & 20,39 & 19,82 & 59,79 & 100 \\
Humedad (\%) & 43,8 & 45,2 & 37,6 & 40,37 \\
Aceite (\% bs) & 23,66 & 0,7 & & $\begin{array}{c}\text { a) } 5,28^{*} \\
\end{array}$ \\
& 5,3 & 3,15 & 3,6 & \\
Proteína (\% bs) & 53,1 & 8,2 & 46,8 & 39,3 \\
Fibra (\% bs) & 1,6 & 1,6 & 1,5 & 1,6 \\
Cenizas (\% bs) & 32,5 & 38,9 & 48 & 43,01 \\
\hline Carbohidratos (\% bs) & & & & \\
\hline
\end{tabular}

* Contenido de aceite total en el fruto fresco teniendo en cuenta solamente el aceite de la pulpa

** Contenido de aceite total en el fruto fresco índices en niveles inferiores si se mejora el proceso de extracción de aceite.

Color, aroma y sabor. El aceite de milpesos «virgen» o crudo posee un color amarillo claro translúcido y brillante, aroma agradable y suave, y sabor perceptible. La apariencia general es como la del aceite de milpesos refinado.

Potencial de uso de aceite de milpesos. Las características fisicoquímicas del aceite de Jessenia batana y sus usos tradicionales permiten conocer la importancia del aceite y determinar su utilidad como aceite de masa, como base para la refinación de cosméticos, en la industria farmacéutica, como lubricante, para curar enfermedades respiratorias dado su contenido de escualeno y vitaminas.

Propiedades físicas, componentes y análisis proximal de los frutos de milpesos. En la Tabla 3 se muestran las propiedades físicas. Se determinó que los frutos de milpesos son drupáceos, con un peso de $15,47 \mathrm{~g}$, presentando un mínimo de $11,16 \mathrm{~g}$ y un máximo de 21,03 g. Asimismo, la semilla (endocarpio) representa el 59,8\% del total del peso, con un promedio de $8,99 \mathrm{~g}$ y sus valores oscilan entre $51,4 \%$ mínimo y un máximo de $65,4 \%$.

Los mayores porcentajes de mesocarpio (Tabla 3) se observan en frutos que están muy cercanos al promedio del peso $(15,47 \mathrm{~g})$. De igual manera, los grupos de frutos con mayor peso de semillas (endocarpio), son los que poseen menor cantidad de mesocarpio y por ende menor cantidad de aceite. Los frutos con menor proporción de cáscara (epicarpio) son los que poseen menor cantidad de mesocarpio.

Análisis proximal. En la Tabla 4 se presenta el análisis proximal.

Proceso de extracción tradicional de aceite de palma de milpesos. La extracción del aceite de palma de milpesos está sujeta a diversas costumbres y tradiciones culturales de las comunidades; sin embargo, en el área de estudio este proceso se realiza fundamentalmente en diez pasos, así:

Cosecha. En este territorio la cosecha es realizada por los aserradores o cazadores que al pasar por una determinada zona se encuentran una palma cargada con frutos (palmas entre $20 \mathrm{~m}$ y $30 \mathrm{~m}$ de altura) y los cosechan; los métodos empleados para esta actividad son la palanca, subirse a un árbol cercano y en la mayoría de casos la tumba de la palma, práctica que es recriminada por los dinamizadores de la vereda, que hasta el momento han logrado concientizar a muchos pobladores. Los frutos listos a cosechar son de color negro intenso; sin embargo, antes de tumbar el racimo realizan una prueba de madurez a los frutos, que consiste en probar con la boca la dureza del mismo (se tiene como referencia $10 \mathrm{~kg}$ de fruto maduro). Lavado. Una vez pesados los frutos de milpesos se lavan con abundante agua limpia para garantizar las condiciones de asepsia.

Madurado o escaldado. Para la maduración de los frutos 
se utiliza agua tibia a más o menos $80^{\circ} \mathrm{C}$ de temperatura, que se deposita sobre los frutos de milpesos. Luego se deja en reposo de 1-2 horas. La cantidad de agua empleada para la maduración depende de la cantidad de frutos a procesar.

- Macerado o amasado. Este procedimiento consiste en estrujar con las manos los frutos de milpesos de tal forma que la corteza (mesocarpio) quede separada de la semilla (endocarpio); para este proceso también se usa un molinillo. La corteza se exprime y se extrae lo que tradicionalmente se denomina leche. Este proceso tiene una duración de casi $10 \mathrm{~min}$. Luego la leche extraída se deja en reposo de 12-24 horas.

- Calentamiento de la leche. La leche obtenida se somete a cocción, de esta forma el aceite inicia a flotar encima de la leche.

- Separado del aceite. Una vez el aceite empieza a verse sobre la leche se va sacando con la ayuda de un cucharón y se deposita en otro recipiente. Este proceso se repite hasta que ya no salga más aceite.

Refritado. El aceite que se obtiene se somete a calentamiento, con el fin de eliminar los residuos de agua (leche) que aún queden. Este proceso demora entre 5 y 10 minutos, dependiendo de la cantidad de aceite.

- Enfriado. El aceite extraído se deja en reposo para que enfríe.

- Cernido. Al dejar el aceite en reposo, las partículas suspendidas en él a causa de la fritura, se van asentando, lo que permite mayor claridad en el aceite. Con la ayuda de un cedazo se cierne el aceite permitiendo que quede más claro y puro.

Embasado. Luego se empaca en frascos de vidrio o plástico y se almacena a temperatura ambiente.

En la Tabla 5 se presenta evaluación de la técnica tradicional de extracción de la palma milpesos. El análisis de los diversos parámetros permite conocer realmente la eficiencia del método de extracción artesanal. El rendimiento de la extracción de aceite es bajo (2,5\%), en valor de $47 \%$ del contenido en el fruto $(5,3 \%)$. Se concluye la necesidad de disponer de otro método que mejore la extracción. La utilización de agua es muy alta, en detrimento de las condiciones ambientales, se requieren 3,24 litros de agua $/ \mathrm{kg}$ de frutos y 139 litros/kg de aceite. Por ello es necesario disminuir al máximo la utilización de agua mediante un método que evite algunas de las etapas, como el colado y el cocinado o que permita una aplicación más eficiente de este recurso.

El consumo de leña es otro factor de gran deterioro ambiental, pues se utiliza $0,9 \mathrm{~kg} / \mathrm{kg}$ de frutos y $37,8 \mathrm{~kg} / \mathrm{kg}$ de aceite. De esta forma, si por ejemplo se producen por este método diez toneladas de aceite por año, se consumirían 378 toneladas de leña en ese mismo tiempo. Para evitar el consumo de leña es necesario modificar este método de extracción por otro que minimice los gastos de combustible y aplicar un tipo de hornilla que permita más eficiencia en la transferencia de calor, así como también revisar los implementos utilizados para estas labores.

El método tradicional tiene una alta demanda de trabajo, sobre todo en las labores de macerado y colado. Esta situación hace necesaria la implementación de modificaciones al proceso de extracción para aprovechar de manera más efi-

Tabla 5

Evaluación de la técnica tradicional de extracción de palma milpesos

\begin{tabular}{lc}
\hline \multicolumn{1}{c}{ Variable } & Cantidad \\
\hline Leña utilizada & $9 \mathrm{~kg}$ \\
\hline Volumen de agua & 32,4 litros \\
\hline Tiempo de madurado o escaldado & 1.5 horas \\
\hline Tiempo de maceración de los frutos y reposo de la leche extraída & 18 horas \\
\hline Tiempo promedio para el calentamiento de la leche & 0,4 hora \\
\hline Tiempo promedio de separación del aceite & 1 hora \\
\hline Tiempo promedio de refritado & 0,17 hora \\
\hline Tiempo promedio de enfriado & 2 horas \\
\hline Tiempo promedio utilizado para cernir (dos pasadas) & 0,8 hora \\
\hline Tiempo total promedio requerido para la obtención de aceite & 23,87 horas \\
\hline Número de personas & 2 \\
\hline Cantidad de materia prima & $10 \mathrm{~kg}$ \\
\hline Cantidad de aceite extraída & $410,25 \mathrm{~cm}^{3}$ \\
\hline
\end{tabular}


ciente el trabajo humano. Teniendo en cuenta la ineficiencia en la extracción de aceite de la palma de milpesos en las comunidades objeto de estudio y su exagerado gasto de leña, mano de obra y agua, esta investigación ha permitido identificar otros métodos para mejorar este proceso artesanal, que es necesario evaluar para determinar el más apropiado para la zona de estudio.

Métodos para la extracción de aceite. Las características de los procesos físicos (prensado) y químicos (solvente) para la extracción de aceite se presentan en la Tabla 6.

Evaluación de los dos métodos descritos. Los resultados de la evaluación de los dos métodos comparados se muestran en la Tabla 7.

\section{MÉTODO PARA MEJORAR LA EXTRACCIÓN DE ACEITE DE LA PALMA DE MILPESOS}

Cosecha. En la cosecha de los frutos de milpesos el principal factor es que no se tale la palma, porque ello implica un aprovechamiento insostenible del recurso. Las formas mediante las cuales se pueden cosechar los frutos son la utilización de una palanca (con un elemento de enganche o filoso para tumbar el racimo), subiéndose a un árbol cercano o si es el caso desde el suelo; otra forma es la utilización de una escalera cuando se considere apropiado; también se puede utilizar un sistema de cuerda con pretales (este sistema consta de un par de pretales, un

\section{Tabla 6 \\ Características de los procesos físicos (prensado) y químicos (solvente) para la extracción de aceite}

\begin{tabular}{|c|c|}
\hline Proceso químico (solvente) & Proceso físico (prensado) \\
\hline \multicolumn{2}{|c|}{ Productos } \\
\hline $\begin{array}{l}\text { La harina o torta tiene un residual de hexano. El porcenta- } \\
\text { je es mínimo pero se puede considerar como un contami- } \\
\text { nante. La harina se puede almacenar por mucho tiempo. }\end{array}$ & $\begin{array}{l}\text { La harina o torta contiene un gran parte del aceite. Se } \\
\text { puede estimar que alrededor de } 20 \% \text { del aceite queda en } \\
\text { la torta con esta tecnología. La harina tiene por este } \\
\text { motivo muy poco durabilidad. }\end{array}$ \\
\hline $\begin{array}{l}\text { Se trata de un proceso considerado como químico y por } \\
\text { este motivo a su vez se toma con algo poco natural. }\end{array}$ & $\begin{array}{l}\text { El aceite se denomina aceite por extracción en frío. Hay } \\
\text { gente que considera este aceite más sano del aceite } \\
\text { extraído por solvente. }\end{array}$ \\
\hline $\begin{array}{l}\text { Es el producto común que siempre tiene el precio más } \\
\text { accesible. }\end{array}$ & $\begin{array}{l}\text { Los productos se pueden a menudo colocar en el mercado } \\
\text { a un precio mayor. }\end{array}$ \\
\hline
\end{tabular}

\section{Eficiencia}

Es un proceso muy eficiente con producción continua. La separación entre aceite y proteína etc. es casi 100\%.
Es un proceso poco eficiente. La extracción está alrededor de $80 \%$ del aceite disponible en la materia prima.

\section{Costo inversión}

Se trata de una inversión muy grande. Una vez instalada la planta difícil se puede desarmar y usar para otros fines.
El costo de inversión es moderado. Para una empresa que arranca en el rubro puede ser una buena idea. Las máquinas siempre se pueden desarmar, transportar y instalar en otro lugar.

\section{Costo operativo}

Los gastos operativos son menores una vez que está en marcha la planta. Se trata de una instalación totalmente automatizada con poca intervención del personal y con procesos bien ajustados con relación de consumo de insumos y energía.
La instalación precisa siempre la intervención del personal por el desgaste de las partes y el consumo de energía es alto. 
Tabla 7

\section{Resultados de factores evaluados para cada método de extracción}

\begin{tabular}{lccc}
\hline \multirow{2}{*}{ Factor } & Ponderación (\%) & \multicolumn{2}{c}{ Valoración } \\
\cline { 3 - 4 } & & Alternativa 1 & Alternativa 2 \\
\hline Ambiental & 25 & 4 & 3 \\
Social y cultural & 25 & 4,225 & 2,89 \\
Económico & 25 & 3,5 & 4 \\
Técnico - Operativo & 25 & 3,865 & 4,09 \\
Total & 100 & 15,59 & 13,98 \\
\hline
\end{tabular}

Alternativa 1: Método físico (con despulpado mecánico y extracción de aceite por prensado) Alternativa 2: Método químico (utilización de solvente)

cinturón de seguridad, una correa abrasadora y una cuerda auxiliar para la correa y adicionalmente requiere de un arco de sierra para el corte de los racimos). Para el uso de los pretales se debe tener en cuenta lo siguiente:

- El pretal más largo va en la superior del estipe y al lado derecho.

- El cinturón de seguridad va después y el pretal más corto va más abajo y al lado izquierdo del escalador.

- Los pretales se suben en forma escalonada, primero el derecho y luego el izquierdo.

- Los pretales no deben estar a más de $10 \mathrm{~cm}$ de separación de la parte media del estipe.

- Al llegar al punto de anclaje para manipular la sierra de arco, se debe hacer el cambio del cinturón de la parte media a la parte superior y amarrar la correa abrasadora con la cuerda auxiliar anexa al cinturón.

- Para el descenso la correa abrasadora siempre va en la parte superior y los pretales más abajo, corriendo primero el izquierdo y luego el derecho en forma escalonada.

- De acuerdo con las habilidades del escalador, este puede bajar del estipe libre de pretales abrazándolo, pero sin soltar la correa abrasadora del cinturón de seguridad.

- El escalador siempre debe manejar como mínimo una cuerda auxiliar amarrada al cinturón de seguridad para izar las herramientas de trabajo como la sierra de arco.

- El escalador debe utilizar cascos de protección y guantes.

- Acondicionamiento. Es muy importante realizar un lavado adecuado de los frutos, de ello depende en gran parte la calidad del aceite. Los frutos poseen una capa cerosa a la que se adhiere polvo y desechos de descomposición orgánica propios de la palma. Se debe utilizar agua limpia, sin ningún tipo de detergente, esparcir los frutos en un recipiente grande de fondo plano y con la ayuda de una escoba y en presencia de agua agitar la masa de frutos, hasta observar la limpieza de estos, que se tornan brillantes. Hacer recambio de agua y volver a agitar. Luego almacenar los frutos en un lugar fresco y proceder lo más rápido posible al escaldado. El agua a utilizar en lavar los frutos no es más de dos veces la porosidad de los frutos (35\%). Para lavar $1 \mathrm{~kg}$ de fruto se debe utilizar un litro de agua.

- Escaldado. Este proceso tiene por objetivo inactivar las enzimas lipásicas o lipolíticas y ablandar los frutos para facilitar el despulpado. Una temperatura de $65^{\circ} \mathrm{C}$ es suficiente para inactivar la acción enzimática, pero para coagular sustancias nitrogenadas y musilaginosas se requieren temperaturas mayores, así también para conseguir un reblandecimiento del fruto que facilite el posterior despulpado.

- Despulpado. En los frutos de milpesos el aceite extraíble está en la pulpa y la cáscara; en la semilla su contenido es muy bajo, por ello es necesario eliminar la semilla. Para extraer el aceite se propone el uso de una despulpadora.

Cocción en seco o fritura. Para obtener la mayor cantidad de aceite por prensado, es necesario que la torta o pulpa posea una temperatura de $90^{\circ} \mathrm{C}$ para facilitar la fluidez del aceite, un determinado contenido de agua y que la estructura celular esté lo más dañada posible para facilitar la migración del aceite. Esto se logra mediante la adición de calor, por cocción o por fritura. La cocción en seco consiste en recalentar la pulpa proveniente del despulpado a $100^{\circ} \mathrm{C}$ durante 30 minutos en un recipiente abierto (olla). La fritura consiste en freir la pulpa en aceite del mismo tipo, durante un tiempo determinado y a una temperatura adecuada, este proceso no requiere de tanto tiempo, puede ser entre 5 y 10 minutos a una temperatura mayor de $100^{\circ} \mathrm{C}$. La cocción resulta muy práctica y de fácil manejo, aunque sea más demorado y consuma más combustible, por eso se presenta como alternativa principal con respecto a la fritura. Para evitar que la pulpa se queme en la parte baja del recipiente de calentamiento es necesario agitarla periódicamente (cada cinco minutos). Al comparar la etapa de cocinado con la cocción en seco, se reduce el consumo de leña, porque el tiempo de exposición al calor se reduce significativamente y 


\section{Bioetnia Volumen 8 No 2 (julio-diciembre), 2011}

la masa a calentar se reduce a una tercera parte, pues no se le adiciona agua como en el método tradicional. Si se implementa un nuevo tipo de hornilla se lograría reducir aún más el consumo de leña. Con la fritura el consumo de leña sería muy bajo, menos del $2 \%$ del consumo en el cocinado tradicional.

Prensado. Este método se implementa porque aumenta el rendimiento en la extracción de un $47 \%$ a un $92 \%$, obteniendo $96 \%$ más de aceite y elimina así el ineficiente paso del cocinado del método tradicional. También trae como segundo beneficio la no utilización de la leña y la disminución de trabajo físico. Se propone el uso de una prensa tipo jaula sencilla.

- Filtrado y fritado. El aceite extraído en la prensa contiene agua e impurezas propias del mesocarpio del fruto, que pasan debido a las altas presiones a que es sometida la torta, por ello es necesario en primera instancia separar el agua, que se observa formado fase, el aceite en la parte superior y el agua en la parte baja. Luego el aceite se lleva a fritado para eliminar la humedad que pueda aún tener y elevar su temperatura para aumentar la fluidez y permitir un posterior filtrado en una tela fina.

Almacenamiento. Se utilizan recipientes de vidrio o plástico debidamente esterilizados y con una tapa adecuada que realice un sellado hermético. El aceite se debe conservar en un lugar fresco, ojalá no expuesto a la luz, así permanecería hasta dos años sin rancearse. Con el método antes expuesto, el consumo de leña total es de $0,2 \%$ del utilizado por el método tradicional, es decir el consumo de leña por $\mathrm{kg}$ de frutos en el proceso se ve reducido de $0,90 \mathrm{~kg}$ a $0,145 \mathrm{~kg}$, representando grandes toneladas en un año. En este sentido, este método propone bajar el consumo de agua de $3,47 \mathrm{l} / \mathrm{kg}$ de frutos a $1.4 \mathrm{l} / \mathrm{kg}$, pues solamente se utiliza en lavar y escaldar los frutos. Asimismo, la relación agua/aceite baja de $139 \mathrm{l} / \mathrm{kg}$ a $19 \mathrm{l} / \mathrm{kg}$ o menos.

\section{CONCLUSIONES}

En Colombia y especialmente en el Pacífico colombiano, las investigaciones que se realizan sobre la palma de milpesos son escasas, lo que dificulta aprovechamiento de la misma, en beneficio de las comunidades rurales de Buenaventura y de toda la región pacífica. Sin duda alguna, la palma de milpesos es una especie promisoria, porque de la misma se pueden aprovechar diferentes partes como tallo, hojas, frutos y raíces, siendo la extracción de aceite un aprovechamiento que puede ayudar a mejorar las condiciones socioeconómicas de las comunidades estudiadas.

Con base en los resultados de esta investigación, se determina que en el sistema tradicional el rendimiento de la extracción de aceite es bajo (extrae alrededor del 47\%), por lo que se hace necesario implementar cambios con técnicas y/o tecnologías más eficientes.

Teniendo en cuenta los factores ambientales, socioculturales, económicos y técnico-operativos la investigación realizada aporta un nuevo método para mejorar la extracción de aceite de la palma de milpesos; sin embargo, a futuro, se deben desarrollar investigaciones que conduzcan a implementar esta nueva forma de extracción de aceite.

\section{LITERATURA CITADA}

Arango, J. U. 2006. Proyectos de seguridad alimentaria desde una perspectiva de gestión ambiental en territorios de comunidades Embera del Atrato medio antioqueño (noroccidente de Colombia). Trabajo de grado (Magíster en Medio Ambiente y Desarrollo). Medellín: Universidad Nacional de Colombia, Facultad de Minas. p. 152.

Arboleda, N. 2008. La palma africana en el Pacífico colombiano: su ilegalidad, consecuencias y violación de derechos territoriales. Luna Azul. 27: 14.

Aguilar, Z. 2005. Influencia de las comunidades Huaorani en el estado de conservación de oenocarpus bataua (arecaceae) en la Amazonía ecuatoriana. Trabajo de grado (Magíster en conservación y gestión del medio natural). Ciudad: Universidad Internacional de Andalucía. p. 100.

Aguirre, R., T. Peske. 1992. Manual para beneficio de semillas. Cali: Centro Internacional de Agricultura Tropical (CIAT). p. 50.

Arias, G. M., M. Castañeda. 1987. Obtención de aceite de Seje. Tecnologia. 167: $35-47$

Arias, J.C., D. Cárdenas. 2007. Manual de identificación, selección y evaluación de oferta de productos forestales no maderables. Bogotá: Instituto Amazónico de Investigaciones Científicas (Sinchi). p. 32.

Balick, M. J. 1992. Jessenia y Oenocarpus. Palmas aceiteras tropicales dignas de ser domesticadas. Roma: FAO. p. 230.

Balick, M. J. 1979. Economic Botany of the Guahibo. I palmae. Economic Botany 33 (4): 361-7.

Corporación para el Desarrollo Industrial de la Biotecnología y Producción. S.f. Evaluación de las variedades más promisorias para la producción de aceite vegetal y su potencial implementación en Colombia. Bogotá: Corporación para el Desarrollo Industrial de la Biotecnología y Producción. p. 131.

Díaz J. A., L. M. Ávila. 2002. Sondeo del mercado mundial de aceite de Seje (Oenocarpus bataua). Bogotá: Instituto de Investigación de Recursos Biológicos Alexander Von Humboldt. p.19.

Hurtado de Barrera, J. 2000. Metodología de la investigación holística. 3a ed. Caracas: Servicios y proyecciones para America Latina. p. 628.

Hurtado de Barrera, J. 2002. El proyecto de investigación holística. Bogotá, Servicios y proyecciones para America Latina. p. 134

Instituto Amazónico de Investigaciones Científicas. 2007. Manual de identificación, selección y evaluación de oferta de productos forestales no maderables. Documento técnico. Bogotá: Instituto Amazónico de Investigaciones Científicas. p. 32.

Miranda, J., F. Montaño, F. Zenteno, H. Nina, J. Mercado. 2008. El Majo (Oenocarpus bataua): una alternativa de biocomercio en Bolivia. La Paz: TRÓPICO-PNBS-FAN. p. 100.

Medina, R. 1999. Nuevo sistema de extracción del aceite de la palma de seje jessenia bataua (Mart) Burret en Cubará. Trabajo de grado (Ingeniero Agrícola). Palmira: Universidad Nacional del Colombia. p. 191.

Pérez, M. A. 2008. Los agrocombustibles: ¿solo canto de sirenas? En: Censat Agua Viva. Agrocombustibles: llenando tanques, vaciando territorios. Colombia; $88 \mathrm{pp}$.

Ramírez, C., L. Gloria. 2003. Palma africana: oportunidad o amenaza para el ambiente y para el país. Gestión y ambiente. 6: 13.

Universidad del Tolima. 2008. Estudio de las palmas presentes en los bosques secundarios del bajo Calima, en tres tipos de bosques en la vereda Las Brisas, Buenaventura, Valle del Cauca. Informe de investigación. Ibagué: Universidad del Tolima. p. 42. 